\title{
Incontinência Urinária em Idosos: um relato de experiência de um ciclo de palestras
}

\author{
Urinary Incontinence in Elderly: \\ an experience report of a lectures cycle
}

\author{
Gabriela Gonçalves Pereira da Silva' • Edmundo de Drummond Alves Junior ${ }^{2}$ \\ Alessandra Conceição Leite Funchal Camacho ${ }^{3}$ Tauan Nunes Maia ${ }^{4}$
}

\begin{abstract}
RESUMO
O presente estudo tem como objetivo relatar a experiência vivenciada durante um ciclo de palestras sobre incontinência urinária direcionada a idosos. Trata-se de um estudo descritivo, do tipo relato de experiência de um ciclo de palestras sobre incontinência urinária realizado em um programa de prevenção de quedas na cidade de Niterói, RJ, Brasil.As palestras ocorreram entre os meses de maio e junho de 2016; totalizando oito encontros divididos em quatros eixos e; com a participação de 68 idosos. Como resultado da observação, análise e discussão dos comentários e dinâmicas propostas durante o ciclo de palestras, constatou-se que os idosos deixam de relatar incontinência urinária por falta de esclarecimento sobre a temática e/ou por constrangimento. As conclusões deste relato apontam para a necessidade de atuação voltada à promoção da saúde de idosos incontinentes, como o estímulo à prática de exercícios físicos específicos para assoalho pélvico e o esclarecimento sobre sintomas e possíveis tratamentos; para a importância do olhar atento e da ação cuidadosa dos profissionais da saúde diante de seus usuários, especialmente idosos; e a adoção da educação em saúde como potencial estratégia de promoção da saúde.
\end{abstract}

Palavras-chave: Envelhecimento; Incontinência Urinária; Promoção da Saúde.

\begin{abstract}
The present study aims to report an experience during a lectures cycle about urinary incontinence targeted at the elderly. This is a descriptive study of the experience report of a lectures cycle about urinary incontinence, performed in a fall prevention program in the city of Niterói, RJ, Brazil.The lectures occurred between May and June 2016; totaling eight meetings divided into four axes and; with a participation of 68 elderly people.As a result of the observation, analysis and discussion of the comments and dynamics proposed during the lectures cycle, it was found that the elderly no longer report urinary incontinence due to lack of clarification on a thematic and/or embarrassment. As conclusions of this report points to the need for action aimed at promoting the health of incontinent elderly people such as stimulating the practice of specific physical exercises for pelvic floor and clarification on symptoms and treatments; for the importance of the look and the careful action of health professionals in front of their users, especially the elderly; and the adoption of health education as a potential health promotion strategy.
\end{abstract}

Keywords: Aging; Urinary Incontinence; Health Promotion.

'Professora de Educação Física (UFF). Especialista em Saúde do Idoso (HUAP/UFF). Mestranda do Programa Acadêmico em Ciências do Cuidado em Saúde (PACCS/UFF). E-mail: silva.ggp@gmail.com. Autor correspondente.

2Professor de Educação Física (UFRJ). Diploma de Estudos Aprofundados (Dea) Histoire Civilisations et Sociétés - Université Rennes 2 Haute Bretagne. Mestrado e Doutorado em Educação Física. Professor da Universidade Federal Fluminense (UFF).E-mail: edmundodrummond@uol.com.br.

${ }^{3}$ Enfermeira. Professora Adjunta da Escola de Enfermagem Aurora de Afonso Costa da Universidade Federal Fluminense (EEAAC/UFF). Docente do Mestrado e Doutorado do Programa Acadêmico em Ciências do Cuidado em Saúde da EEAAC/UFF. Doutora em Enfermagem pela Escola de Enfermagem Anna Nery (UFRJ). E-mail: cicacamacho@gmail.com.

4Professor de Educação Física (UFF). Especialista em Terapia Intensiva (HUAP/UFF). Mestre em Ciências do Cuidado em Saúde (PACCS/UFF). E-mail: taunum@ hotmail.com. 


\section{INTRODUÇÃO}

O processo de envelhecimento populacional é um fenômeno constatado e tende a progredir significativamente no Brasil e no mundo. Através da associação entre a diminuição das taxas de natalidade, aumento da expectativa de vida e diminuição da taxa de fecundidade ${ }^{(1)}$, estima-se que a população mundial de idosos seja de 2 bilhões até $2050^{(2)}$, e que este público represente $13,67 \%$ da população em 2020 no Brasil( ${ }^{(1)}$. Este processo associado à alteração do perfil epidemiológico ${ }^{(3)}$, pode aumentar as demandas individuais e coletivas para a área da saúde, como: o crescimento do número de doenças crônicas não transmissíveis, dos custos de procedimentos e das hospitalizações ${ }^{(1-4)}$. Trata-se de um processo gradual, resultado da interação entre os elementos cronológicos, biológicos, psicológicos e sociais ${ }^{(5)}$.

Apesar disto, "o modelo de declínio biológico ainda predomina"(6). Há, por exemplo, modificações importantes na anatomia e fisiopatologia do trato urinário inferior que alteram diretamente os órgãos e tecidos envolvidos no processo de micção ${ }^{(5)}$. Com o passar dos anos, a bexiga tem a capacidade vesical e contratibilidade do músculo detrusor reduzidas, e volume residual e hiperatividade aumentados; a uretra reduz sua pressão de fechamento e; a próstata aumenta de volume. Também são observados eventos importantes, como: o aumento da produção noturna de urina, a redução da produção de estrógeno na mulher e o aumento da incidência de infecções recorrentes ${ }^{(5)}$. Estes fatores favorecem 0 aparecimento da incontinência urinária (IU), condição muito observada entre os idosos.

Entendida como "qualquer perda involuntária de urina" (7), a IU acomete homens e mulheres em todas as faixas etárias, entretanto, o sexo feminino e a idade avançada são considerados fatores de risco ${ }^{(5-8)}$. Além destes, outros estudos ${ }^{(8-10)}$ apontam como fatores de risco: obesidade, paridade, vias do parto, menopausa, cirurgias ginecológicas, alterações prostáticas, constipação intestinal, tabagismo, histórico familiar, traumas do assoalho pélvico, raça, consumo de cafeína, exercícios intensos na região abdominal, hipertensão arterial, Diabetes Mellitus e Doença de Parkinson. Em idosos, a prevalência estimada de IU varia entre $17 \%$ e $31 \%$ em homens, e $23 \%$ e $63 \%$ entre as mulheres ${ }^{(5-9-11-12)}$, valores que podem alterar de acordo com o perfil da população e local de realização dos estudos.

A classificação dos tipos de IU é feita de acordo com os sintomas relatados pelos pacientes, podendo ser de três tipos: de esforço, quando está relacionada a esforços que promovam aumento da pressão abdominal (como tossir, rir, espirrar); de urgência, quando acompanhada ou procedida de urgência miccional e; mista, quando ocasionadas tanto por esforço quanto por urgência ${ }^{(5-9)}$.
Ela é um fator de risco importante para quedas, principalmente por fatores como a IU de urgência e a noctúria. As conseqüências dos eventos de queda em idosos são graves e, se associadas aos episódios de perda de urina, podem agravar o estado de isolamento social e depressão; além de desestimular a ingesta hídrica, diminuir os cuidados com a higiene pessoal e o aumentar os eventos de infecção urinária ${ }^{(5)}$.

A IU está diretamente ligada à diminuição da qualidade de vida do indivíduo, causando isolamento social, dependência e depressão(13) e, por vergonha ou constrangimento, é pouco relatada pelos idosos, o que dificulta o tratamento precoce dos sintomas ${ }^{(8)}$

Este estudo tem, então, como objetivo relatar a experiência vivenciada durante um ciclo de palestras sobre IU direcionada a idosos.

\section{MÉTODO}

O presente estudo é um relato de experiência de um ciclo de palestras com a temática da IU, realizado no programa de extensão intitulado "Prev-Quedas: prevenindo as quedas agora, evitará que o próximo a cair seja você" (14), desenvolvido no Instituto de Educação Física da Universidade Federal Fluminense (UFF).

Como critério para participação no programa, o interessado deve apresentar atestado médico atualizado que comprove autorização para praticar exercícios físicos e ter sua ficha de cadastro devidamente preenchida no momento da inscrição.Atualmente, o projeto atende gratuitamente 167 indivíduos da cidade de Niterói, RJ, Brasil. Os participantes são indivíduos fisicamente ativos, com idade entre 25 e 89 anos - apesar de aproximadamente $70 \%$ ter 60 anos ou mais -, $60 \%$ deles possuem nível médio ou superior completo e $80 \%$ mora acompanhado por cônjuge ou familiar. As comorbidades mais prevalentes entre eles são a hipertensão e a diabetes.

As intervenções do projeto são realizadas numa perspectiva intergeracional de promoção da saúde ${ }^{(15)}$, nas quais são compreendidas as demandas do processo multifatorial do envelhecimento ${ }^{(14)}$. As oficinas acontecem duas vezes por semana no turno da manhã e da tarde, totalizando nove turmas com limite de vinte alunos em cada uma delas. São oferecidas oficinas de força, consciência corporal e ginástica. Seu planejamento é baseado não somente na sistematização de exercícios físicos, mas na prática do lazer através de experiências lúdicas e prazerosas, que estimulem a socialização e aproximação com os elementos da cultura popular brasileira e com as experiências de vida dos alunos ${ }^{(14)}$.

Após incorporação de exercícios específicos para assoalho pélvico durante algumas aulas oferecidas pelo projeto em 2016, o tema da IU passou a ser algo recorrente nas indagações dos alunos. Logo, a escolha do 
tema e a idealização do ciclo de palestras emergiram em função desta crescente demanda dos participantes do projeto, que apresentavam questionamentos acerca do conceito, etiologia e possíveis tratamentos para incontinência urinária. As abordagens à professora responsável pela oficina eram cada vez mais freqüentes, e ocorriam de forma individual após o horário das aulas e sempre com muita descrição, o que culminou na urgência para a realização do ciclo de palestras.

O ciclo de palestras foi elaborado por uma equipe multiprofissional motivada pela vontade de compreender a relação dos idosos com os possíveis sintomas e queixas de perda de urina e, direcionar informações sobre a temática através da educação em saúde em um espaço lúdico, interativo e prazeroso. A equipe foi composta por uma enfermeira, um nutricionista e uma professora de educação física, mediadora da vivência. Todos os membros estavam no $2^{\circ}$ ano da Residência em Saúde do Idoso pelo Programa de Residência Multiprofissional do Hospital Universitário Antônio Pedro (HUAP/UFF) e atuavam no projeto há pelo menos I ano.

O ciclo consistiu de oito palestras vivenciadas às segundas-feiras dos meses de maio e junho de 2016, nos turnos da manhã e da tarde, e com duração de sessenta minutos cada encontro.A divulgação do ciclo de palestras ocorreu oralmente no horário das oficinas do projeto e com cartazes espalhados pelo campus da universidade.

Dos 72 idosos interessados no ciclo de palestras, 68 participaram de todos os encontros. Dentre os ausentes, 03 disseram não ter disponibilidade de horário e 01 relatou não ter mais o interesse em participar. Totalizaram, então, 03 homens e 65 mulheres com idade entre 60 e 88 anos.

Em reunião composta pela equipe multiprofissional, as palestras foram organizadas em quatro eixos, com dois encontros cada um. Estes seguiam uma seqüência gradativa de aproximação com a temática, partindo do conhecimento do todo (o corpo humano) e chegando à compreensão da IU, suas características e particularidades. Assim sendo, os eixos buscaram seguir uma sequência didática que partia de conhecimentos mais simples para o mais complexo, sendo ordenados da seguinte forma: "Conhecendo o nosso corpo", com exposição de conceitos gerais do corpo humano e seu funcionamento;"Por onde sai o meu xixi?", com o objetivo de direcionar os conhecimentos sobre a anatomia do assoalho pélvico e do mecanismo de micção; "Vazou, e agora?", um bloco de palestras voltado para a orientação sobre a classificação, fatores de risco, sintomas, caracterização da perda da urina e possíveis tratamentos e; "Chega de dúvidas!", último bloco de palestras, cujo qual os participantes explanariam possíveis dúvidas sobre a temática e seus autorrelatos.
Durante os encontros, foram utilizados como materiais didáticos: figuras, slides, vídeos, cartazes e bonecos. A organização das cadeiras em forma de círculo, a elaboração de dinâmicas interativas e a utilização de um vocabulário acessível, pretendiam estimular a interação entre a palestrante e os participantes, a fim de diminuir o possível desconforto quanto à explanação da temática, já que ela é socialmente entendida como um tabu social ,e pouco é falada ${ }^{(8)}$.

O presente estudo seguiu as recomendações do Conselho Nacional de Saúde segundo a Resolução n. ${ }^{\circ}$ 466/ I2, e tem aprovação do Comitê de Ética e Pesquisa da Faculdade de Medicina do HUAP/UFF, sob o parecer n. ${ }^{\circ} 851.371$.

\section{RESULTADOS E DISCUSSÃO}

Ao longo do ciclo de palestras, observou-se que o envolvimento dos participantes aumentou gradativamente. Nos primeiros encontros, a participação ativa nas dinâmicas em grupo foi menor quando comparados aos encontros finais. Muitos pareciam envergonhados e relatavam prever um ciclo de palestras com conteúdos exclusivamente expositivos. A partir do terceiro encontro (segundo eixo), os idosos expuseram-se mais através de perguntas e relatos pessoais, o que foi interpretado como uma aparente diminuição do desconforto sobre a temática. Foi possível eliminar dúvidas e relatar experiências diárias de perda de urina através da naturalização de termos e expressões relacionadas ao processo de micção e evacuação, poucos faladas no cotidiano, como "xixi", "fazer xixi na calça", entre outras; e do estímulo à participação nas atividades coletivas.

A vergonha parece como uma forte justificativa de omissão dos sintomas de IU. Durante o ciclo de palestras foi estabelecida pelos participantes uma relação entre temas como perda de urina, declínio de capacidades físicas, aumento de limitações, uso de fraldas e perda de dependência e autonomia. Sob esta perspectiva, aproximase 0 indivíduo incontinente de problemas psicossociais importantes que alteram substancialmente sua qualidade de vida pela perda da autoestima, alteração na vida sexual, aparecimento de sintomas depressivos e isolamento social(5-8-16-18).

Observou-se que a maioria dos participantes demonstrava não compreender muito bem a relação de seus órgãos com o mecanismo de micção. Algumas idosas, por exemplo, mostraram-se surpresas ao constatarem que a eliminação da urina acontece pela uretra e não pelo canal vaginal, como imaginavam. Neste sentido, a escolha pela utilização de uma linguagem clara e inteligível foi primordial para uma melhor compreensão dos conteúdos pelos participantes. $O$ trabalho com a comunidade e o fato de estar diante de um grupo heterogêneo, sugere a sociali- 
zação de informações de maneira acessível e que facilite a compreensão pelos usuários( ${ }^{(8)}$. Baseado nos conhecimentos sobre IU adquiridos pelo pelos idosos, torna-se possível direcionar ações em saúde com autonomia e consciência diante das implicações decorrentes desta e de outras doenças ${ }^{(8-15)}$.

Os participantes também relataram a falta de esclarecimento sobre o conceito da IU e seus possíveis tratamentos. Estes associavam esta patologia ao uso de protetores (como absorventes e fraldas geriátricas), como se a falta de necessidade de utilizá-los descaracterizasse a doença. Estudos ${ }^{(8-13-16-18)}$ apontam que a falta de conhecimento dos sintomas de IU desestimula a procura por tratamentos especializados, o que associado à falta de esclarecimento sobre tratamentos e possíveis intervenções para prevenção e eliminação dos sintomas, dificulta o seu diagnóstico precoce.

O tratamento para IU deve ser multiprofissional, envolvendo um planejamento adequado para cada tipo de incontinência e priorizando o tratamento não farmacológico, seguido do farmacológico e, em última instância, o procedimento cirúrgico ${ }^{(5)}$. Apesar das recomendações, os tratamentos não invasivos, como a prática de exercícios para o fortalecimento do assoalho pélvico, são pouco divulgados pelos profissionais de saúde, que incentivam prioritariamente tratamentos medicamentosos e cirúrgicos ${ }^{(17)}$.

Outro elemento observado foi a naturalização da IU, entendida pelos idosos como parte do processo de envelhecimento e inerente às idades mais avançadas. Relatos que naturalizam a IU foram constantes durante a vivência. Este pode ser mais um dos motivos que inibem os relatos de perda de urina e afastam os indivíduos da procura por tratamento ${ }^{(8-13-16-18)}$. Apesar de um terço da população relatar sintomas de IU, apenas $50 \%$ dela procura os serviços de saúde por este motivo ${ }^{(8)}$.

Além destas, outras causas podem ser apontadas como justificativas para o subdiagnóstico de IU, tais como: medo e desconforto em serem examinadas ${ }^{(17)}$; possível adaptação a esta condição na fase inicial dos sintomas ${ }^{(18)}$; o fato de priorizarem o tratamento e controle de comorbidades tidas como mais graves e comuns ao envelhecimento, como hipertensão e diabetes ${ }^{(17)}$; e a falta de conhecimento dos profissionais da saúde sobre fatores associados ${ }^{(8)}$.

Ações efetivas de promoção da saúde têm se tornado cada vez mais importantes, especialmente na atenção básica. Como uma estratégia factível, a educação em saúde, veiculada por meio das instituições de saúde, ensino e comunicação em massa, possibilita a troca de informações e instruções aos indivíduos, estimulando a prevenção e detecção precoce de patologias, e o controle de fatores de risco pela mudança no estilo de vida ${ }^{(17-19)}$. Para os idosos, é necessário que espaços de educação em saúde estejam cada vez mais palpáveis, através da utilização de tecnologias acessíveis e lúdicas para facilitar o emponderamento do usuário e proporcionar tomada de decisões sobre sua saúde de maneira autônoma e consciente ${ }^{(15)}$. Diante do cenário descrito, percebe-se que as relações estabelecidas no indivíduo com IU extrapolam as alterações fisiológicas do enveIhecimento, envolvendo também os aspectos psicológicos, conjugais e sociais diretamente ligados a ela, e a maneira com que ela é percebida pelos idosos. Neste sentido, o trabalho multidisciplinar tem papel fundamental na prevenção e tratamento da $I^{(12)}$, na medida em que considera as múltiplas dimensões do indivíduo e, conjuntamente, elabora estratégias para contribuir para melhoria da qualidade de vida ${ }^{(17)}$.

\section{CONCLUSÃO}

O envelhecimento populacional gera demandas no Brasil e no mundo. Por isso, os serviços de saúde devem direcionar suas ações para a prevenção e tratamento de patologias associadas ao envelhecimento.A IU é uma patologia constante em idosos e, apesar de não estar associada diretamente a causa de morte, traz conseqüências significativamente negativas para a vida dos idosos, como a depressão e $o$ isolamento social. Dentre os fatores que dificultam o diagnóstico precoce de IU e inibem a procura por tratamentos, foram apresentados: a vergonha, a falta de esclarecimento sobre a caracterização da doença e possíveis ações preventivas e tratamentos.

O ciclo de palestras relatado neste estudo foi utilizado como estratégia de educação em saúde, e é entendido como um facilitador na troca de informações e empoderamento dos usuários.

As conclusões deste estudo apontam para a necessidade da atenção direcionada não só aos idosos alunos do Projeto Prev-Quedas, mas aos usuários do serviço público de saúde através de exercícios de fortalecimento da musculatura de assoalho pélvico, que visem eliminar os agravos da IU. Sugere-se também que a educação em saúde seja utilizada em larga escala como importante estratégia de promoção da saúde, visando o esclarecimento e direcionamento de ações específicas sobre prevenção, tratamento dos sintomas de IU e melhoria da qualidade de vida. Ressalta-se também a importância do olhar atento e ação cuidadosa dos profissionais da saúde diante de seus usuários, especialmente idosos.

Como limitação, o ciclo de palestras teve um número restrito de participantes por questões estruturais, como o tamanho e disponibilidade das salas. Neste sentido, novos estudos, que envolvam um número maior de participantes, se fazem necessários para pensar em novas estratégias de promover a saúde de idosos com IU. 


\section{REFERÊNCIAS}

I. Ervatti LR, Borges GM, Jardim AP. Mudança demográfica no Brasil no início do século $\mathrm{XXI}$ : subsídios para as projeções da população. Rio de Janeiro: Instituto Brasileiro de Geografia e Estatística [Internet]. 2015 [acesso em 02 ago 2017]. Disponível em: https://biblioteca.ibge.gov.br/visualizacao/livros/liv93322.pdf.

2. Organização das Nações Unidas (ONU). O mundo terá dois bilhões de idosos em 2050; OMS diz que 'envelhecer bem deve ser prioridade global' [Internet]. 2014 [acesso em 02 ago 2017]. Disponível em: http://sbgg.org.br/wp-content/ uploads/20 I5/I0/OMS-ENVELHECIMENTO-20 I 5-port.pdf.

3. Santos GS, Escudeiro CL, Souza WL, Santos LM, Silva VC, Santo FHE. Implantação do programa hospital amigo do idoso: relato de experiência. Rev Enf Atual [Internet]. 2017 [acesso em 04 ago 2017]; 20(3):47-52. Disponível em: http:// inderme.com.br/revistas/revista_18-07.pdf.

4. Silva JVF, Silva EC, Rodrigues APRA, Miyazawa AP. A relação entre o envelhecimento populacional e as doenças crônicas não transmissíveis: sério desafio de saúde pública. Cadernos de graduação: ciências biológicas e da saúde [Internet]. 2015 [acesso em 04 jan 20I8]; 2(3):91-100. Disponível em: https://periodicos.set.edu.br/index.php/fitsbiosaude/article/ view/2079//268.

5. Maciel AC. Incontinência Urinária. In: Freitas V, Py L. Tratado de Geriatria e Gerontologia. 3 ${ }^{\mathrm{a}}$. ed. Rio de Janeiro: Guanabara Koogan; 2012.

6. Farinatti PTV. Teorias do Envelhecimento. In Farinatti PTV. Envelhecimento, promoção da saúde e exercício: tópicos especiais em aspectos biológicos e psicossociais. I ${ }^{\text {a }}$ ed. Barueri: Manole; 2013.

7. Abrams P, Cardozo L, Fall M, Griffiths D, Rosier P, Ulmsten U, Van Kerrebroeck P, Victor A, Wein A. Standardisation SubCommittee of the International Continence Society: the standardisation of terminology of lower urinary tract function: report from the standardisation sub-committee of the International Urology Continence Society. Urology [Internet]. 2003 [acesso em 03 ago 20 I7]; 6I (I):37-49. Disponível em: http://www.goldjournal.net/article/S0090-4295(02)02243-4/pdf.

8. Bolina AF, Dias FA, Santos NMF, Tavares DMS. Incontinência Urinária Autorreferida em idosos e seus fatores associados. Rev RENE [Internet]. 2013 [acesso em 04 ago 2017]; 14(2):354-63 Disponível em: http://www.revistarene.ufc.br/ revista/index.php/revista/article/view/909.

9. Nardi AC et al. Urologia Brasil. I ${ }^{\mathrm{a}}$. ed. Rio de Janeiro: Sociedade Brasileira de Urologia [Internet]. 2014 [acesso em 04 ago 20I7]. Disponível em: http://sbu-sp.org.br/wp-content/ uploads/2016/02/Livro_Diretrizes_Urologia.pdf.

10.Araujo MP, Parmigiano TR, Negra LGD, Torelli L, Carvalho CG, Wo L, Manito ACA, Girão MJBC, Sartori MGF. Avaliação de Assoalho Pélvico de Atletas: existe relação com incontinência urinária? Rev Bras Med Esporte [Internet]. 2015 [acesso em: 03 ago 20I7]; 2 I(6):442-46. Disponível em: http://www.scielo.br/pdf/rbme/v2 In6/I5 I 7-8692-rbme-2 I-06-00442.pdf.

I I.Mourão LF, Luz MHBA, Marques ADB, Benício CDAV, Nunes BMVT, Pereira AFM. Caracterização e fatores de risco de incontinência urinária em mulheres atendidas em uma clínica ginecológica. Rev Estima [Internet]. 2017 [acesso em 04 jan 2018]; I5(2):82-9I. Disponível em: https://www.revistaestima.com.br/index.php/estima/article/view/352/pdf.

12.Silva KC, Ferreira E, Alves R. Avaliação da prevalência de incontinência urinária em idosas através do questionário de impacto de incontinência urinária (ICIQ-SF). Revista Amazônia Science \& Health [Internet]. 2014 [acesso em 04 jan 20 I8]; 2(2):44-8. Disponível em: http://ojs.unirg.edu.br/index. $\mathrm{php} / 2 /$ article/view/485/252.

I3.Marques LP, Schneider IJC, Giehl MW, Antes DL, Orsi E. Fatores demográficos, condições de saúde e hábitos de vida associados à incontinência urinária em idosos de Florianópolis, Santa Catarina. Rev Bras Epidemiol [Internet]. 2015 [acesso em 03 ago 2017]; 18(3):595-606. Disponível em: http://www.scielosp.org/pdf/rbepid/v18n3/pt_I4I5-790X-rbepid-18-03-00595.pdf.

I4.Farinatti PTV, Guimarães JMN, Alves Junior ED. Envelhecimento e quedas: fatores de risco e prevenção. In: Farinatti PTV. Envelhecimento, promoção da saúde e exercício: tópicos especiais em aspectos biológicos e psicossociais. $\mathrm{I}^{\mathrm{a}}$. ed. Barueri: Manole; 2013.

15.Farinatti PTV. Promoção da saúde e envelhecimento: conceitos, definições e princípios. In Farinatti PTV. Envelhecimento, promoção da saúde e exercício: tópicos especiais em aspectos biológicos e psicossociais. I a ed. Barueri: Manole; 2013.

16.Carvalho PM. Risco de quedas em mulheres idosas com incontinência urinária na comunidade [Dissertação]. Viseu: Instituto Politécnico de Viseu [Internet]. 2013 [acesso em 04 ago 20I7]. Disponível em: http://repositorio.ipv.pt/handle/l0400.19/1973.

17. Henkes DF; Fiori A, Carvalho JAM, Tavares O, Frare JC. Incontinência urinária: o impacto na vida de mulheres acometidas e o significado do tratamento fisioterapêutico. Semina Cienc Biol Saúde [Internet]. 2015 [acesso em 03 ago 2017]; 36(2):45-56. Disponível em: http://www.uel.br/revistas/uel/ index.php/seminabio/article/view/21746/I7952.

18.Dellu MC. Incontinência urinária no climatério: prevalência, fatores associados e impacto na qualidade de vida [Tese] São Paulo: Universidade de São Paulo [Internet]. 2015 [acesso em 05 ago 2017]. Disponível em: http:// www.teses.usp.br/teses/disponiveis/6/6 I36/tde-191020I5I00242/pt-br.php.

19.Mallmann DG, Neto NMG, Sousa JC, Vasconcelos EM. Educação em saúde como principal alternativa para promover a saúde do idoso. Ciênc Saúde Colet [Internet]. 2015 [acesso em 02 set 20 I7]; 20(6): I763-72. Disponível em: http://www. scielo.br/pdf/csc/v20n6/|4| 3-8| 23-csc-20-06- | 763.pdf. 